\title{
FOSILIZACIÓN FONOLÓGICA EN EL INGLÉS DE UNA MUESTRA DE PROFESORES DE INGLÉS COMO SEGUNDA LENGUA
}

\author{
Bonnie Brown \\ Berta Flores
}

\begin{abstract}
RESUMEN
En este artículo se analiza la fonología del interlenguaje en una muestra de habla de diecinueve profesores de inglés como segunda lengua en la Escuela de Lenguas Modernas de la Universidad de Costa Rica, con el fin de descubrir los errores fonológicos fosilizados tanto a nivel segmental como prosódico. Las desviaciones grupales en los niveles fonémico y prosódico parecen ser el efecto de una constante transferencia negativa de patrones fonológicos del Español. Los errores intralinguales (generalización de reglas fonológicas del Inglés) son menos frecuentes.
\end{abstract}

\begin{abstract}
The phonology in the interlanguage of a group of Costa Rican university professors of English as a second language is analyzed to discover phonological errors through the application of error and contrastive analyses. Group deviances at the phonemic and prosodic levels appear to be the effect of constant negative transfer of Spanish phonological patterns. Intralingual errors (generalization of English phonological rules) are less frequent.
\end{abstract}

\section{Introducción}

En este artículo se analiza la fonología del interlenguaje en una muestra de habla de diecinueve profesores de inglés como segunda lengua en la Escuela de Lenguas Modernas de la Universidad de Costa Rica, con el fin de descubrir los errores fonológicos fosilizados tanto a nivel segmental como prosódico.

Así mismo se analizan las posibles causas de esta fosilización partiendo de las teorías de adquisición de un segundo idioma que tratan el problema de la fosilización en el habla de personas que iniciaron la adquisición de L2 (segunda lengua de aquí en adelante) durante o después de la pubertad.

El problema de la fosilización de estructuras incorrectas en el interlenguaje ha sido discutido entre otros por Schuman (1978), Corder (1976), Selinker y Lamendella (1979), 
Beebe (1980), Krashen (1985), Omaggio (1986), Ellis (1986) y Tarone (1987). Estos lingüistas coinciden en describir el fenómeno de la fosilización como la estabilización, en el interlenguaje, de formas incorrectas que conllevan la aparición de lo que se denomina acento extranjero.

\section{Metodología}

\subsection{Los hablantes}

Se establecieron dos criterios para determinar la población:

a. Que fueran profesores en propiedad.

b. Que su lengua materna fuera el español.

El grupo de diecinueve sujetos incluye a cuatro varones y quince mujeres cuyas edades oscilan entre treinta y cuatro y cincuenta y cinco años. Los sujetos iniciaron su aprendizaje del inglés a una edad promedio de diecinueve años (sin tomar en cuenta los años de estudio del inglés en los colegios públicos). Cinco de los informantes participaron en programas de intercambio de un año antes de iniciar sus estudios universitarios. El noventa y uno por ciento de la población ha realizado estudios universitarios en inglés en los Estados Unidos. El promedio de años de estudio en ese país es de 2,98 años. En total los sujetos de estudio han dedicado un promedio de 8,91 años al estudio del inglés, 5,93 en Costa Rica y 2,98 años en los Estados Unidos.

La población ha trabajado en la Universidad de Costa Rica un promedio de 15,68 años. Han enseñado cursos en todos los niveles de la carrera de inglés y en todas las áreas: gramática, pronunciación, composición, etc.

\subsection{Extracción de los datos}

Se reunieron los hablantes durante dos horas en grupos de tres o cuatro para discutir un tema relacionado con la docencia y se recogió el corpus con cámara filmadora de video. El uso de la cámara contribuyó a que los participantes usaran un estilo cuidadoso y no el estilo libre que es más sistemático. Sin embargo, el estilo cuidadoso es, en este caso, representativo del habla de los profesores en el aula.

El corpus recogido se transcribió ortográficamente en su totalidad. Luego, empleando el método de análisis de errores sugerido por Corder en 1974 se identificaron, describieron y clasificaron los fonemas y factores prosódicos fosilizados. El análisis de los fonemas se realizó en su contexto lingüístico; es decir, se tomó en cuenta el efecto de los sonidos adyacentes al fonema fosilizado, la posición del mismo en la sílaba y en la palabra, y el efecto de aspectos prosódicos tales como la acentuación. Para tal efecto se hicieron transcripciones fonéticas usando el Alfabeto Fonético Internacional. El análisis de los factores prosódicos se llevó a cabo tanto en la palabra aislada (en el caso de la acentuación) como en la frase (acentuación, ritmo y entonación). Aspectos sintácticos relevantes fueron tomados en consideración cuando el caso lo ameritaba. 
Cada investigadora identificó los errores independientemente y posteriormente se compararon los resultados. Cuando hubo discrepancias se escuchó de nuevo la grabación y aquellos errores en cuya identificación no hubo acuerdo se descartaron. El paso siguiente consistió en describir y clasificar los errores de acuerdo a los procesos fonológicos empleados por los sujetos para lidiar con el problema: substitución, eliminación, epéntesis, asimilación progresiva y regresiva, sonorización y desonorización de fonemas. Se analizó también la cantidad relativa de las vocales (duración normal de las vocales) pues se consideró un factor determinante en la identificación de la consonante sonora posterior y por ende, en la claridad del mensaje. En el caso de los factores prosódicos se clasificaron los errores de acuerdo al acento de intensidad (especialmente el que obedece a circunstancias históricas por cuanto constituye uno de los rasgos más característicos que distingue a los dos idiomas), también se analizó el acento rítmico en la frase y la entonación en el grupo fónico.

En el proceso de análisis se empleó la siguiente simbología:

$$
\begin{array}{ll}
\longrightarrow & =\text { se convierte en } \\
\mathrm{V} & =\text { vocal } \\
\mathrm{C} & =\text { consonante } \\
\bar{I} & =\text { posición del fonema } \\
\# \quad & =\text { en el contexto } \\
\overline{\#} & =\text { posición inicial en la palabra } \\
\overline{\mathrm{CC}} & =\text { posición final en la palabra } \\
\mathrm{CCC} & =\text { conjunto de dos consonantes } \\
\varnothing & =\text { eliminación } \\
\overline{ } & =\text { acento }
\end{array}
$$

Con el fin de marcar la diferencia entre equivocación y error, se registraron como errores aquellas desviaciones fonológicas que un mismo informante repitió un mínimo de tres veces durante la conversación. Cuando un mismo error lo produjo un mínimo del $20 \%$ de la población, se tomó como desviación de grupo.

Finalmente, se analizaron las posibles causas de los errores: transferencia negativa de la lengua materna y procesos intralinguales (sobregeneralización y aplicación incompleta de patrones de la segunda lengua). El análisis contrastivo constituyó un valioso instrumento en este proceso.

\section{Resultados}

Las tablas 1 y 2 muestran los porcentajes de desviación de grupo por transferencia negativa a nivel de vocales y consonantes y los procesos fonológicos empleados por los sujetos. 


\subsection{Desviaciones de grupo}

\subsubsection{Errores por transferencia negativa a nivel segmental}

Tabla 1

Vocales

Procesos fonológicos

\begin{tabular}{|c|c|c|c|c|}
\hline & Substitución & & Falta de reducción & $\begin{array}{l}\text { Falta } \\
\text { de Alargamiento }\end{array}$ \\
\hline$|\mathfrak{x} / \rightarrow| \mathrm{e} /$ & $|a| \rightarrow|0|$ & $|a| \rightarrow / o /$ & $\left\{\begin{array}{l}\{/ \mathrm{i} /\} \rightarrow / \mathrm{i} /, / \mathrm{e} /, / \mathrm{o} /, / \mathrm{e} / \\
\{/ \mathrm{a} /\}\end{array}\right.$ & 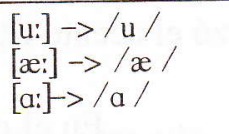 \\
\hline $52,6 \%$ & $42,1 \%$ & $21 \%$ & $26,3 \%$ & $26,3 \%$ \\
\hline
\end{tabular}

Tabla 2

Consonantes

\begin{tabular}{cc}
\hline \multicolumn{3}{c}{ Procesos fonológicos } \\
\hline Desonorización & Eliminación \\
\hline & CC $\rightarrow$ C $\varnothing$ \\
& CC $\rightarrow$ CC $\varnothing$ \\
& CCC $\rightarrow \varnothing C C$ \\
$21 \%$ & CCC $\rightarrow$ C $\varnothing \mathrm{C}$ \\
& $52,6 \%$ \\
\hline
\end{tabular}

La tabla 3 muestra la desviación de grupo a nivel prosódico por transferencia negativa. La acentuación fue el mayor problema tanto a nivel de palabra como a nivel de frase.

\subsubsection{Errores por transferencia negativa a nivel prosódico}

Tabla 3

Acentuación

\begin{tabular}{c|ccc}
\hline A nivel de palabra & \multicolumn{2}{c}{ A nivel de frase } \\
\hline Sílabas posteriores & Verbo "ser/estar" & Pronombres & Auxiliares \\
$31,5 \%$ & $31,5 \%$ & $42,1 \%$ & $31,5 \%$ \\
\hline
\end{tabular}


La tabla 4 refleja la desviación de grupo por errores intralinguales. Aunque se dan otros errores a nivel segmental y prosódico, la única desviación de grupo es la que presenta la tabla 4.

\subsubsection{Errores intralinguales}

Tabla 4

Vocales

\begin{tabular}{c} 
Vocales \\
\hline Proceso fonológico \\
Substitución \\
$/ \mathrm{ij} / \rightarrow / \mathrm{I} / \mathrm{o} / \mathrm{i} / \rightarrow / \mathrm{ij} /$
\end{tabular}

$36,8 \%$

\section{Conclusiones}

La literatura sobre la teoría de la transferencia apunta hacia la poderosa influencia que ejercen la fonética y la fonología de la lengua materna sobre la pronunciación de una segunda lengua. El análisis contrastivo ha sido hasta ahora herramienta común para describir las dificultades que los estudiantes de un segụndo idioma tienen debido a la transferencia negativa de patrones de su lengua nativa. Es por esto que siguiendo la sugerencia de Brière (1968) citado en Odlin (1990: 113) hemos hecho una comparación lingüística cruzada de los patrones fonéticos y fonológicos del inglés y del español con el fin de sacar algunas conclusiones. Debemos de reconocer, sin embargo, que la transferencia no es el único factor que interfiere, pues factores tipológicos y universales que algunas veces operan independientemente de la transferencia y otras veces junto con ella, son también responsables de la imprecisión en la pronunciación de una segunda lengua.

\subsection{Errores por Transferencia Negativa}

Las variaciones de grupo más notorias, en cuanto a pronunciación de fonemas vocales se refiere, se dieron en el cambio de los fonemas /æ/, / a /, y / / / en sílaba acentuada. El fonema / æ/ se substituyó por un sonido con las características del fonema / e / del español. El fonema / a / del inglés se articuló con las características del sonido / o / del español y el fonema / ə/ se pronunció también como el fonema / o / del español. En las tres substituciones, el denominador común es el aumento en el grado de tensión muscular y el movimiento hacia una posición posterior. Cabe hacer la observación de que la escogencia de la vocal española podría haber sido inducida por la ortografía de la palabra. Veamos algunos ejemplos: 


$\begin{array}{ll}\text { [ənว́ðər] } \rightarrow \text { [ənóðər }] & \text { another } \\ {[\text { hæ:v }] \longrightarrow[\text { hef] }} & \text { have } \\ {[\text { prá:bləm }] \rightarrow[\text { próbləm }]} & \text { problem }\end{array}$

Los sonidos / I / y / a / de las vocales reducidas son especialmente víctimas de la transferencia negativa del español. Debemos recordar que aunque en español las vocales inacentuadas se relajan y se les da un timbre menos definido y preciso en el lenguaje rápido y familiar, no llegan en ningún caso al grado de vaguedad e imprecisión que se manifiesta en las vocales relajadas del inglés (Navarro 1972: 177). En este estudio, los sonidos / I / y / a / fueron frecuentemente sustituidos por las vocales / i /, / o / y / e / del español. Es importante destacar que la falta de interiorización del patrón de reducción de las vocales inacentuadas resulta en un marcado acento extranjero, especialmente por la alta frecuencia con que ese patrón se repite en la cadena hablada del inglés.

Cabe señalar también que el discurso más lento y enfático de los sujetos contribuyó a que los sonido / i / y / a / fueran substituidos por vocales más claras y distintas del español.

Otra desviación importante a nivel vocálico es la falta de alargamiento de la vocal acentuada seguida de consonante sonora; no obstante, tal problema no sorprende por cuanto las vocales españolas en la conversación corriente son siempre breves. Existe en español una semejanza cuantitativa entre vocales fuertes y débiles, y si se compara esa cantidad relativa con la duración normal de las vocales largas en otros idiomas "puede decirse que en la pronunciación ordinaria española no hay vocales propiamente largas" (Navarro 1972: 199). No es de extrañar, entonces, que los sujetos de estudio presenten problema para alargar vocales.

En cuanto a desviaciones de grupo en la pronunciación de consonantes, encontramos que en conjuntos de dos y tres consonantes en posición final de palabra se recurrió a la eliminación de alguno de los segmentos para simplificar la estructura de la sílaba.

El acento es el primer factor prosódico que analizaremos en la palabra, para posteriormente analizarlo en la frase. Para comprender mejor la transferencia negativa del patrón de acentuación del español al inglés que los sujetos de estudio en general hacen, resumiremos lo que Alarcos expresa en su Fonología Española (1976: 202). Dice Alarcos que en español no puede haber más de una sílaba culminativa en cada palabra y la posición de esa sílaba culminativa, la cual posee función distintiva, "varía, es libre; pero esta libertad está limitada a las tres últimas sílabas de la palabra". La acentuación de la palabra en español se representa entonces de la siguiente manera:

_ _ _, _ _ ó _ _ _ . Los casos de palabras con acento en otras sílabas lo constituyen aquellas palabras compuestas en cuyo caso cada componente conserva su acento propio: inútilmente, contratiempo, etc.

También asegura Alarcos (1976: 204) que "fonológicamente, sólo tiene pertinencia en español el contraste entre sílaba acentuada y sílaba átona" pues los llamados acentos secundarios no tienen más relevancia que la de matizar en un sentido u otro lo que se dice. Su único valor es, pues, expresivo. Todo esto podría explicar la tendencia generalizada entre los sujetos de estudio a acentuar las palabras en alguna de las tres últimas sílabas, lo cual contradice el patrón de acentuación del inglés, en el cual, según Nash (1977: 42), normalmente 
se acentúan las palabras en sílabas anteriores a la sílaba acentuada de la misma palabra en español. Ejemplos de errores cometidos constantemente durante el discurso de los sujetos son:

coordinátor, evaluáted, hesitáte, commentáry, vocabuláry.

De acuerdo a Nash, los sustantivos compuestos por dos palabras en inglés se acentúan en el primer componente mientras que, como ya explicamos anteriormente, en español cada palabra guarda su propio acento. No es de extrañar entonces que los informantes recurrentemente acentuaran sustantivos compuestos en sus dos componentes, por ejemplo:

\section{báckgróund, ánythíng, feédbáck, etc.}

En cuanto a la acentuación en la frase debemos destacar que tanto en inglés como en español existe una estrecha relación entre el acento de intensidad y la función sintáctica que la palabra desempeña. En ambas lenguas (aparte de razones psicológicas, lógicas, fisiológicas o rítmicas) se acentúan normalmente los verbos, adverbios, sustantivos y adjetivos. La inacentuación, en cambio, se da en palabras como artículos, preposiciones y conjunciones; sin embargo el carácter histórico ligado a la tradición lingüística del español permite que palabras con función sintáctica secundaria sean acentuadas en la frase. Los verbos auxiliares, por ejemplo, se acentúan todos, de ahí que deduzcamos que el error de acentuar los auxiliares modales como en la frase: I would guarantee that he... lo mismo que los auxiliares do y have es un claro ejemplo de interferencia. Esta transferencia negativa se da también en la acentuación constante del verbo $b e$, al punto de que constituye una desviación de grupo. La frecuente acentuación de los pronombres sin que se dé una circunstancia especial que lo amerite (establecer un contraste, por ejemplo) parece reflejar una fuerte transferencia negativa del sistema de acentuación del español al inglés. Al distorsionarse el acento que es la base del ritmo en inglés, se distorsionan también el ritmo y el tono; como consecuencia, se proyectan actitudes que el hablante nativo del inglés interpreta como enfado, exaltación o arrogancia:

\section{I can say what I have heard...}

\subsection{Errores intralinguales}

A aquellos errores cuyo origen no parece ser la transferencia negativa del español, se les buscó su causa dentro del sistema mismo de la segunda lengua.

En el caso de los sonidos vocálicos encontramos que una importante desviación de grupo es la substitución del fonema / i / en sílaba acentuada y en monosílabas por el fonema / ij /. En el caso de palabras polisílabas el error se cometió tanto en posición inicial de palabra, como en input [ijnput], como en posición media de palabra como en activities [æktijvitız]. Para las investigadoras este proceso de substitución de / I / por / ij / constituye un claro ejemplo de sobregeneralización. Pareciera que los sujetos, en un esfuerzo por mejorar la precisión en los sonidos, producen el sonido / ij / en contextos en que / I / es lo correcto. Este fe- 
nómeno resulta interesante por cuanto contradice las predicciones de un análisis contrastivo, puesto que una diptongación de este tipo resulta extraña e inclusive chocante al hablante español. Contrario a lo que sucedió, era de esperar que los informantes pronunciaran el fonema / I / en forma breve y con un timbre invariable de principio a fin. Sin embargo, la tendencia de la laxitud a la tensión podría sugerir una interacción entre transferencia y sobregeneralización.

En cuanto a errores intralinguales en aspectos prosódicos de la lengua, ya anotamos que la acentuación en sílabas anteriores a la antepenúltima en palabras no compuestas resulta extraña al sistema fonológico del español. Deducimos entonces que el origen de este error es más bien una sobregeneralización del patrón de acentuación del inglés. Ejemplos claros de este error son:

commúnication, pronúnciation, cóntamination and óbligation.

\section{Discusión}

Hay evidencia suficiente para inferir que la transferencia negativa de patrones de la lengua materna a nivel segmental y prosódico parece ser la causa principal de la fosilización fonológica en el interlenguaje de los profesores de inglés. Los errores intralinguales, por el contrario, aparecen con menos frecuencia y se dan sobre todo por sobregeneralización de patrones de la segunda lengua. El análisis de los datos refleja que aquellos sujetos cuyo interlenguaje se acerca más a L2 presentan estructuras fosilizadas, principalmente en aspectos prosódicos tales como acento en la palabra y en la frase. De la evidencia se puede inferir que dichas estructuras son producto de la transferencia negativa de patrones de acentuación del español al inglés. Esta evidencia nos lleva a pensar que hay menos conciencia en los sujetos de estudio sobre las reglas y patrones que conforman el área prosódica de la lengua meta. A pesar de que el estilo utilizado en las discusiones de grupo fue cuidadoso, se nota menos control y autocorrección en esta área. En el caso de los sujetos con mayor número y variedad de estructuras fonológicas fosilizadas, resalta la transferencia negativa no sólo en factores prosódicos sino también en fonemas y alófonos. En este caso los errores intralinguales son menos frecuentes y se producen sobretodo por la substitución de una vocal laxa por su contraparte tensa y viceversa.

Los procesos fonológicos preferidos por los sujetos de estudio para dar forma a la fonología de su interlenguaje son la substitución y eliminación de fonemas. En cuanto a la simplificación de conjuntos de consonantes en posición final de palabra, los resultados coinciden con los resultados obtenidos por Flores y Rodríguez (1994: 107) en cuanto a que los sujetos muestran preferencia por el proceso de eliminación.

En un intento por relacionar los resultados obtenidos con la información recabada mediante una encuesta administrada a los profesores, señalamos lo siguiente:

De acuerdo a dicha información los sujetos de estudio iniciaron su aprendizaje del inglés en la pubertad cuando, de acuerdo a Krashen (1985: 13), el filtro afectivo sube en forma dramática. La adolescencia, sugiere Krashen, es una etapa que "inhibe la adquisición 'natural' de la lengua, incluyendo la adquisición de la fonología".

Otro aspecto que podría relacionarse con la fosilización fonológica es la metodología mediante la cual los sujetos iniciaron el proceso de aprendizaje de la segunda lengua. Los 
métodos audiolingual y gramatical (grammar translation) no permiten una comunicación real y limitan la cantidad de información comprensible transmitida.

La encuesta administrada revela también que el output de los profesores es dramáticamente bajo. Los profesores hablan inglés un promedio de 26,30 minutos por día durante el año calendario, principalmente dentro del aula.

El punto anterior nos lleva a deducir que la calidad de input a la que se exponen los sujetos diariamente no es la óptima. Debemos recordar que el interlenguaje de los estudiantes contiene errores que se repiten constantemente y que terminan no sólo ampliando los márgenes de tolerancia de desviaciones del modelo de $\mathrm{L}_{2}$ sino también, en cierta medida, contaminando el interlenguaje del profesor.

\section{Limitaciones}

1) La primera y tal vez más importante limitación es la que Labov (1970:47) llama la "Paradoja del Observador", la cual consiste en que para recoger buenos datos se requiere una observación sistemática. Sin embargo, este tipo de observación no permite llegar hasta el estilo vernacular, que es el más sistemático y por lo tanto el principal objetivo del estudio lingüístico.

2) La segunda limitación es la que menciona Ellis (1987:52) al decir que un análisis de errores examina el interlenguaje en un momento dado y no arroja mucha luz sobre el proceso de desarrollo que han seguido los sujetos.

3) Debido a la metodología empleada de discusión en grupo, fue imposible controlar la cantidad y el tipo de estructuras empleadas por los sujetos. De hecho, algunos profesores se limitaron a hacer comentarios muy cortos, lo cual limitó la posibilidad de análisis. En cada caso se hizo una observación al respecto al pie de los resultados individuales.

4) No se contó con un equipo muy sofisticado para recoger y posteriormente analizar el corpus. Por lo tanto, los resultados reflejan los errores más obvios que captaría un profesional entrenado en detectar problemas fonológicos.

\section{Sugerencias para futuras investigaciones}

Consideramos que esta investigación es un aporte más a la comprensión de la naturaleza de los procesos que caracterizan la fonología del interlenguaje y el fenómeno de la fosilización; sin embargo, es mucho lo que falta por descubrir y describir.

El corpus recogido resulta ser materia prima para futuros trabajos de investigación en el campo de la gramática, semántica, análisis del discurso y trabajos comparativos inglés-español. Además revela ciertas actitudes de los profesores de inglés hacia su profesión, su imagen, el idioma y la cultura norteamericana dignas de ser investigadas. 
Tres estudios específicos que podrían realizarse son:

1) Una investigación exhaustiva sobre la transferencia negativa de los patrones de los rasgos prosódicos del español al inglés.

2) Un estudio semejante al presente con profesores de inglés cuya lengua materna no es el español. Los resultados de este estudio serían muy valiosos para corroborar o descartar la interferencia de la lengua materna en la fonología del interlenguaje de los sujetos.

3) Una investigación sobre la incidencia de procesos fonológicos universales en la conformación de la fonología en el interlenguaje de los profesores de inglés.

\section{Apéndice A: Resumen de errores y ejemplos de desviaciones de grupo}

Transferencia negativa a nivel de fonemas

I. Vocales

A. Substitución

1. $|æ| \rightarrow|\mathrm{e}|$

/ gæps / $\rightarrow$ / geps /

/ æktıvitı / $\rightarrow$ / ektíjvitı /

2. $\mid$ 贯 $/ \rightarrow / 0 /$

/ sam / $\rightarrow$ / som /

/ fonetıks / $\rightarrow$ / fonetrks /

3. $|\mathrm{a} / \rightarrow| \mathrm{o} /$

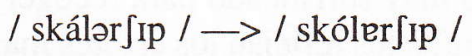

/ pra:bləm / $\rightarrow$ / probləm /

B. Falta de reducción

/ fəsilətejt / $\rightarrow$ / fesilitejt /

/ parsan / $\rightarrow$ / person /

C. Falta de alargamiento

/ mæ:d / $\rightarrow$ / mæt /
/ sa:lvd / $\rightarrow$ / solvd /
/ skuw:l / $\rightarrow$ / skuwl / 
II. Consonantes

A. Desonorización

$$
\begin{aligned}
& \text { / læongwidz / } \rightarrow \text { / læongwis / } \\
& \text { / kampəzifən / } \rightarrow \text { / komposifen / } \\
& \text { / rekəgnajz / } \rightarrow \text { / rekəgnajs / }
\end{aligned}
$$

B. Eliminación

$$
\begin{array}{ll}
\mathrm{CC} \rightarrow \mathrm{C} \varnothing & \text { / saluw } \int ə n z / \longrightarrow \text { / soluw jən / } \\
\mathrm{CCC} \rightarrow \mathrm{CC} \varnothing & \text { / æsks / } \rightarrow \text { / æsk / } \\
\mathrm{CCC} \rightarrow \varnothing \mathrm{CC} & \text { / Ikspiriəns / } \rightarrow \text { / ispiriəns / }
\end{array}
$$

\section{Transferencia negativa a nivel prosódico}

I. Acentuación

A. A nivel de frase

1. Verbos ser/estar

That shouldn't be so

The process is in between.

2. Pronombres

We thought that we needed...

They thought they were...

Can we have cursos de extensión for ourselves?

\section{Auxiliares}

He has exteriorized

I would guarantee that he...

B. A nivel de palabra

1. En sílabas posteriores

a. palabras simples

$$
\text { motivate } \rightarrow \text { motivate }
$$$$
\text { vocabulary } \rightarrow \text { vocabulary }
$$$$
\text { coordinator } \rightarrow \text { coordinator }
$$

b. sustantivos compuestos

$$
\begin{aligned}
& \text { feédbàck } \rightarrow \text { feédbáck } \\
& \text { báckgròund } \longrightarrow \text { báckgróund } \\
& \text { hónor ròll } \longrightarrow \text { hónor róll }
\end{aligned}
$$


Errores intralinguales

I. Substitución

1. $/ \mathrm{ij} / \rightarrow / \mathrm{I} /$

/ spijk / $\rightarrow$ / spik /

/ fijəl / $\rightarrow$ / fil /

/ wijklı / - / wiklı /

2. $/ \mathrm{I} / \longrightarrow / \mathrm{ij} /$

$/$ ðis $/ \rightarrow /$ ðijs /

/ strl / $\rightarrow$ / stijl /

/ bits $/ \rightarrow$ / bijts /

Apéndice B: Causas de la fosilización por autor

1. EDAD

Ervin-Tripp 1974

Selinker and Lamendella 1980

Dulay, Burt and Krashen 1982

Macnamara 1983

Krashen 1985

Ioup 1987

Beebe 1987

2. RESISTENCIA PSICOLÓGICA

Tarone 1978

Hatch 1983

3. TRANSFERENCIA

Krashen 1981, 1985

Dulay, Burt and Krashen 1982

Corder 1983

Schacter 1983

Tarallo 1983

Gass 1984

Ellis 1985

4. FALTA DE CALIDAD Y CANTIDAD DE "INPUT"

Corder 1983

Ellis 1985

Pica 1985

Krashen 1985

Larsen-Freeman 1985 


\section{FILTRO AFECTIVO ALTO}

Dulay, Burt and Krashen 1982

Krashen 1985

6. CAUSAS SOCIO-EMOCIONALES

Gardner 1972

Schumann 1980

Dulay, Burt and Krashen 1982

Savignon 1983

Seliger 1983

Ellis 1985

Snow 1985

7. FALTA DE OPORTUNIDADES PARA PROBAR HIPÓTESIS Ellis 1985

Bley-Vroman 1986

Schacter 1986

\section{ESTABILIZACIÓN EN UN "PLATEAU"}

Selinker and Lamendella 1980

Richards 1989

9. RESTRICCIONES RELACIONADAS CON EL ESTUDIANTE

Alatis 1981

Hansel 1981

Dulay, Burt and Krashen 1982

Beebe 1983

Hatch 1983

Schacter 1986

Ioup 1987

10. FALTA DE NECESIDAD

Corder 1974

Selinker and Lamendella 1980

\section{Bibliografía}

Alarcos, Emilio. 1976. Fonología Española. Madrid: Editorial Gredos, S.A.

Beebe, L. 1987. "Sociolinguistic Variation and Style Shifting in Second Language Acquisition”. En: Ioup, G. y S.H. Weinberger (eds.).

1987. "Myths About Interlanguage Phonology". En: Ioup, G. y S.H. Weinberger (eds.). 
Corder, S.P. 1974. "Idiosyncratic Dialects and Error Analysis". En: Richards, J. (ed.).

1974. “The Significance of Learner's Errors”. En: Schumann, J. and N. Stenson (eds.).

1983. “A Role For the Mother Tongue”. En: Krashen y R. Scarcella (eds.).

Croft, K. (ed.). 1980. Readings on English as a Second Language. MA: Winthrop Publishers, Inc.

Ellis, R. 1987. Understanding Second Language Acquisition. Oxford: Oxford University Press.

Flores B. y X. Rodríguez. 1994. "The Influence of Language Transfer on Consonant Cluster Production”. Revista de Filología y Linguiística de la Universidad de Costa Rica. XX (1): 99-112.

Ioup, G. y S.H. Weinberger (eds.). 1987. Interlanguage Phonology and the Acquisition of a Second Language System. Cambridge: Newbury House Publishers.

Krashen, S. 1980. "The Monitor Model for Adult Second Language Performance". In Croft, K. (ed.).

Krashen, S. y R. Scarcella (eds.). Issues in Second Language Research. Rowley, MA: Newbury House.

Labov, W. 1970. "The Study of Language in its Social Context". Studium Generale. 20, 30-87.

Nash, Rose. 1977. Comparing English and Spanish: Patterns in Phonology and Orthography. New York: Regents Publishing Company.

Navarro, Tomás. 1972. Manual de Pronunciación Española. Madrid: Raycar, S.A.

Odlin, T. 1990. Language Transfer . (2nd ed). Cambridge: Cambridge University Press.

Omaggio, Alice C. 1986. Teaching Language in Context: Profeciency-Oriented Instruction. Boston: Heinle and Heinle Publishers, Inc.

Richards, J. (ed.). 1974. Error Analysis: Perspectives on Second Language Acquisition. London: Longman Group Limited.

Schumann, J. y N. Stenson (eds.). 1974. New Frontiers in Second Language Learning. Mass.: Newbury House Publishers Inc.

Selinker, L. 1974. “Interlanguage.” En: Richards, J. (ed.). 
1979. "The Role of Extrinsic Feedback in Interlanguage Fossilization". Language Learning. 29: 363-75.

Selinker y J.T Lamendella. 1980. "Fossilization in Interlanguage Learning." En: Croft, K (ed.).

Tarone, E. 1987. "Some Influences on the Syllable Structure of Interlanguage Phonology". En: Ioup, G. y S.H. Weinberger.

1987. "The Phonology of Interlanguage". En: Ioup, G.y S.H. Weinberger.

"Systematicity and Attention in Interlanguage". Language Learning. 32, 69-83. 